\title{
STUDY OF THE PERIODICITY OF MOONQUAKES AND THEIR CAUSALITIES
}

\author{
Lyara Villanova Silverio, Elder Yokoyama and George Sand França, Universidade de Brasília - UnB.
}

Copyright 2019, SBGf - Sociedade Brasileira de Geofísica

This paper was prepared for presentation during the $16^{\text {th }}$ International Congress of the Brazilian Geophysical Society held in Rio de Janeiro, Brazil, 19-22 August 2019.

Contents of this paper were reviewed by the Technical Committee of the $16^{\text {th }}$ International Congress of the Brazilian Geophysical Society and do not necessarily represent any position of the SBGf, its officers or members. Electronic reproduction or storage of any part of this paper for commercial purposes without the written consent of the Brazilian Geophysical Society is prohibited.

\begin{abstract}
The Moon is the nearest planetary body to Earth, producing the largest gravitational force. The moonquakes are found to be largely governed by the Earth position (Kolvankar et. al., 2010), it is possible to observe that the peaks of occurrence of total seismic activity on the Moon occur in periods of the main tidal components, although the pattern of occurrence is not related simply to the anomalistic month cycle, but also to perturbations external to the orbit lunar. We present a preliminary analysis of the data recorded by six seismic stations, installed on lunar soil by NASA, in the Apollo missions, characterizing periodicity of moonquakes occurrence with their respective perturbations, correlating cyclicity with geological and orbital mechanisms.
\end{abstract}

\section{Introduction}

The Moon is a celestial body that figures in the mythology of almost all civilizations of the ancient world (Song, 1983). However, it was only from the observations of Galileo Galilei in 1600 that it became scientifically studied. Scientific knowledge about the Moon developed continuously from 1600, having its apex in the twentieth century, during the space missions Apollo. Of the contributions, one of the most relevant was the recording of about 12,000 moonquakes (Nakamura, 2005), for the six stations installed on lunar soil (Figure 1), which were subdivided into shallow, deep, thermal and meteorological impacts. Although the recording and processing of these earthquakes has brought important information about the composition and internal structure of the Moon (eg, Goins et al., 1981; Lognonne et al., 2001; Zhao et al., 2011), some questions about the lunar seismicity are still the subject of debate in the literature, such as their causality and their cyclicity.

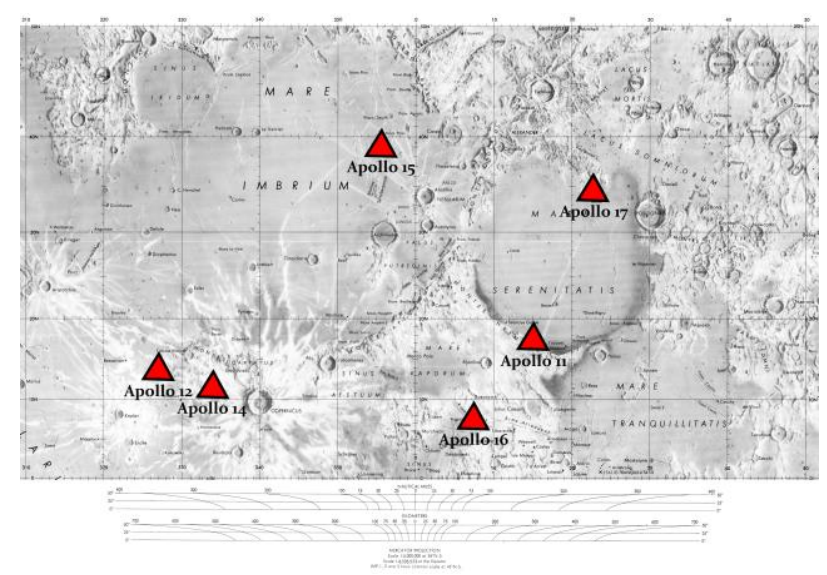

Figure 1 - Landing sites of the Apollo missions.

The earthquakes recorded in the Apollo missions (Figure 2) showed anomalous characteristics compared to terrestrial ones, where: (I) the energy released from most of these events is very small, even the largest ones have energies smaller than the energy of an earthquake of magnitude 3.0 ML on Earth (Toksoz et al., 1977); (II) approximately $99 \%$ of the moonquakes occurring in subsurface are between 750 and $1400 \mathrm{~km}$ deep (Toksoz et al., 1977) and (III) of the 166 delimited deep seismic foci established from the data at the time of arrival, all but one are located on the Moon's nearest Earth side (Gagnepain-Beyneix et al., 2006).

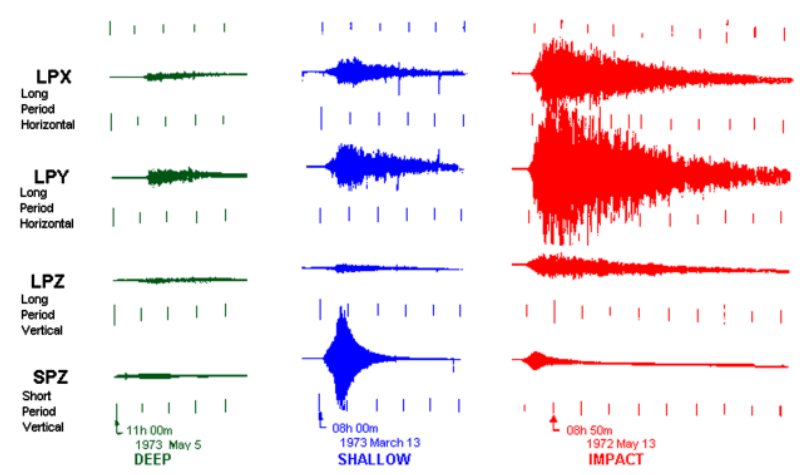

Figure 2 - Signature of typical events observed in the recorded data. 
During the period of operation of the Apollo seismographic stations, the peaks in total activity, taken as the sum of all the events detected at a given station per unit time, occurred at intervals of about 14 days. The most prominent peaks in the spectrum calculated by Latham et. al. (1972) occurred in periods of 14 and 28 days (Figure 3 ) and were associated with tidal effects. In general, peaks in moonquakes activity occur close to apogee and perigee (Kolvankar et al., 2010). However, the pattern of occurrence is not simply related to the monthly changes in the apogee-perigee cycle, but also to other tidal components that arise from disturbances outside the lunar orbit.

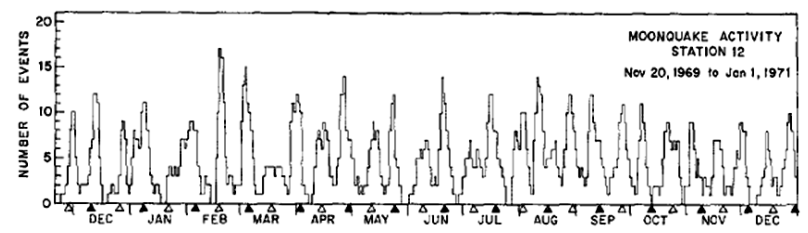

Figure 3 - Number of moonquakes per day recorded in Apollo 12 between November 20, 1969 and January 1, 1971. Black triangles indicate perigee and white triangles indicate apogee. Retrieved from Latham et. al., 1972.

\section{Method}

The collected data, which are made available by NASA and IRIS, of the six seismographic stations installed by the Apollo missions from 1969 to 1977 , which total 12,000 events, were used. First, a seismic catalog, in which each event has been classified and among them was produced by impact of meteorites were discarded, since they are of artificial nature. The study of time series for deep moonquakes, for shallow moonquakes and for thermal moonquakes, focusing on cyclicity, will be carried out by means of spectral (e.g., REDFIT) and Wavelet analysis.

\section{Results}

A "seismic band" can be observed when plotting the epicenters of the recorded moonquakes (Figure 4), and to date it has been possible to ascertain that the natural seismicity of the Moon is correlated with (i) its synodic period, approximately 29.53 days, since the ellipticity of its orbit results in different velocities in the motion of the Moon, when it is closer to or farther from the Earth, generating mass movement due to gravitational attraction; (ii) the inclination of the plane of the Moon's orbit around the Earth in relation to the ecliptic (plane of the Earth's orbit around the Sun. This rotation to the west of the orbital plane of the Moon occurs by the differential force exerted by the Earth's equatorial bulge, caused by the rotation of the Earth) and (iii) the apparent motion of the Moon in relation to the apparent motion of the Sun. This work, although preliminary, serves as an initial reference to the work being developed.

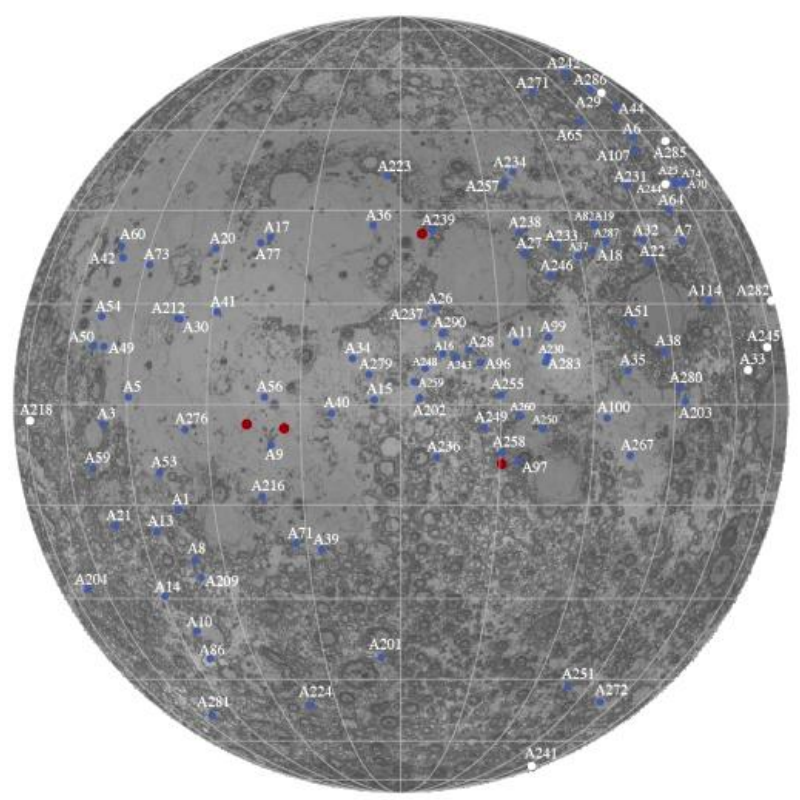

Figure 4 - Map of the near side of the Moon, with epicenters of recorded events plotted. Retrieved from NASA, 2019.

\section{Conclusions}

The proposed analyzes, per hour, allowed to infer the possible mechanisms generating moonquakes, which will greatly facilitate the study of their cyclicity, as well as to refine the understanding of lunar structure and dynamics in later studies. These relationships can be an important point to make the discussion about the understanding of the universe more profound and to encourage studies on the Moon and other planetary bodies.

\section{Acknowledgments}

I am particularly grateful for the assistance given by the Institute of Geosciences and the Seismological Observatory, both from the University of Brasilia, for all technical support and to NASA, for the data available.

\section{References}

Gagnepain-Beyneix, J., Lognonné, P., Chenet, H., Lombardi, D., Spohn, T., A seismic model of the lunar mantle and constraints on temperature and mineralogy. Physics of the Earth and Planetary Interiors 159 (2006) 140-166.

Goins, N., Dainty, A., Toksoz, M., 1981. Lunar seismology: the internal structure of the Moon. Journal of Geophysical Research 86, 5061-5074.

Kolvankar, V. G., Deshpande, S. S., Manjre, A. S., Pansare, S., More, S. and Thakur, N. Lunar Periodicities and Earthquakes. New Concepts in Global Tectonics Newsletter, no. 56, September, 2010. 
Latham, G., Ewing, M., Dorman, J., Nakamura, Y. Lunar Structure And Dynamics- Results From The Apollo Passive Seismic Experiment. 1972.

Lognonne, P., Gagnepain-Beyneix, J., Chenet, H. A new seismic model of the Moon: implications for structure,thermal evolution and formation of the Moon. Earth and Planetary Science Letters 211 (2003) 27-44.

Nakamura, Y., 2005. Farside deep moonquakes and deep interior of the moon. Journal of Geophysical Research 110, E01001.

NASA, Planetary seismology. NASA Marshall Space Flight Center, 2019.

Song, G. A Correlation Between Large-Scale Asymmetry Of The Moon And The Focus Of Moonquakes. University of Manchester, England, 1983.

Toksoz, M., Dainty, A., Solomon, S., Anderson, K., 1977. Structure of the moon. Reviews of Geophysics and Space Physics 12, 539-567.

Zhao, D., Arai, T., Liu, L., Ohtani, E. Seismic tomography and geochemical evidence for lunar mantle heterogeneity: Comparing with Earth. Global and Planetary Change 9091 (2012) 29-36. 